\title{
WAVE OPTICS ANALYSIS OF CAMERA IMAGE FORMATION WITH RESPECT TO RECTANGULAR APERTURE
}

\author{
Abdulkadir Iyyaka Audu ${ }^{1}$ and Abdul Hamid Sadka ${ }^{2}$ \\ Department of Electronic and Computer Engineering, \\ Brunel University, London, United Kingdom
}

\begin{abstract}
In general, analysing cameras is a difficult problem and solutions are often found only for geometric approach. In this paper, the image capturing capability of a camera is presented from optical perspective. Since most compact cameras can acquire only visible light, the description and propagation method of the visible part of the electromagnetic spectrum reflected by a scene object is made based on Maxwell's equations. We then seek to use this understanding in the modelling of the image formation process of the camera. The dependency of camera sensor field distribution on aperture dimension is emphasized. This modelling leads to an important camera and image quality parameter called Modulation Transfer Function. The model presented is based on a wave optics in which the wavefront is modified by the lens after diffraction has taken place at the camera rectangular aperture positioned at the front focal point of the lens. Simulation results are presented to validate the approach.
\end{abstract}

\section{KEYWORDS}

Electromagnetic spectrum, Camera aperture dimension, 2D and 3D sensor field distribution, Modulation transfer function.

\section{INTRODUCTION}

The study of optics is believed to have started with the Sumerians during the 4000 B.C. The discovery of rock crystal lens, Palaeolithic wall paintings often found in caves of almost total darkness and the use of a lamp and a hand-held mirror were significant developments which point to the cultivated high level civilization during that time [1]. The idea about vision is arguably considered to have began in Greece by Euclid and others. Their understanding of vision was that humans see because some rays called eye rays emanate from the eye, strike the scene objects and are returned to the eye.

The second century A.D. witnessed a major conceptual shift when more quantitative experiments started. Refraction of light at air-water interface was studied by Claudius Ptolemy [1].

Conventional imaging, and especially computer-assisted imaging, has brought many areas of research and applications to a mature plateau with astonishing advantages [2]. Therefore, we argue that camera modelling should be primarily directed towards describing mathematically any improvement strategies that can be made to the image formation process, and noise characterisation and evaluation $[3,4]$. 
In a compact (refined) camera, the numerical form of the continuous variation of the scene object being sensed is produced. Comprehensively speaking, the modelling pipeline of the image formation process of a camera consists of radiometry, the camera (optics and sensor), the motion associated with the camera, the processing, the display, and the interpretation as functional blocks [4]. Only the optics and sensor model will be presented based on wave optics and the use of rectangular aperture.

This paper is structured as follows. Section two covers a brief overview of imaging systems. The main idea of the paper is presented in section three. In section four, simulation results are presented and discussed. An overall conclusion about the paper is drawn in section five.

\section{IMAGING SYSTEM IN PERSPECTIVE}

A large number of compact cameras are based on perspective projection model with a single centre of projection. They are generally characterised by a typically narrow field of view (FOV) of $50^{\circ} \times 35^{\circ}$ and hence sometimes called directional cameras. FOV makes compact cameras less popular in certain vision applications. A compact camera is widely used in the acquisition of both static and dynamic scenes. Traditional cameras capture light onto photographic film. Digital cameras use electronics, usually a charge coupled device (CCD) to store digital images in computer memory inside the camera.

Enhancement of FOV is the main reason for the invention of catadioptric imaging systems. A merit of single view point [5] is significant in the face of the complexity arising from the shape, position, and orientation of the reflecting surfaces employed.

Large FOV above $180^{\circ}$ is the main characteristic of omnidirectional cameras. Furthermore, as pointed out in [6], images of all scene points cannot be represented by intersections of camera rays with a single image plane. For that reason, rays of the image are represented as a set of unit vectors in three-dimensional (3D) such that one vector corresponds just to one image of a scene point. Omnidirectional cameras usually find application in areas such as structure from motion, and surveillance where considerably stable ergo_motion computation is expected.

It is stated in [7] that single-camera panoramic stereo imaging systems using an unusual arrangement of curved mirrors, is becoming attractive. Such systems solve the problem of capturing a panoramic image with a single motionless camera, and are also capable of positioning and measuring an object with a single imaging process.

The objects of interest in the world are simply a set of points. The intensity acquisition of these points is achieved through the non-singular transformation capability of cameras which are almost usually based on signal processing principles, measurement, and algorithms [8]. Although important research and developmental effort have been targeted at camera fabrication based on state-of-the art technology, there still exist practical limits on the intrinsic parameters such as focal length, [9]. For example, in Figure 1, the dependence of two important parameters depth of view (DOF) and FOV on camera focal length $\mathrm{f}$, working distance $\mathrm{u}$, magnification , circle of confusion c, f-number of camera lens $f_{n}$, and sensor physical size $s$, are expressed in (1) and (2) respectively[9]. 


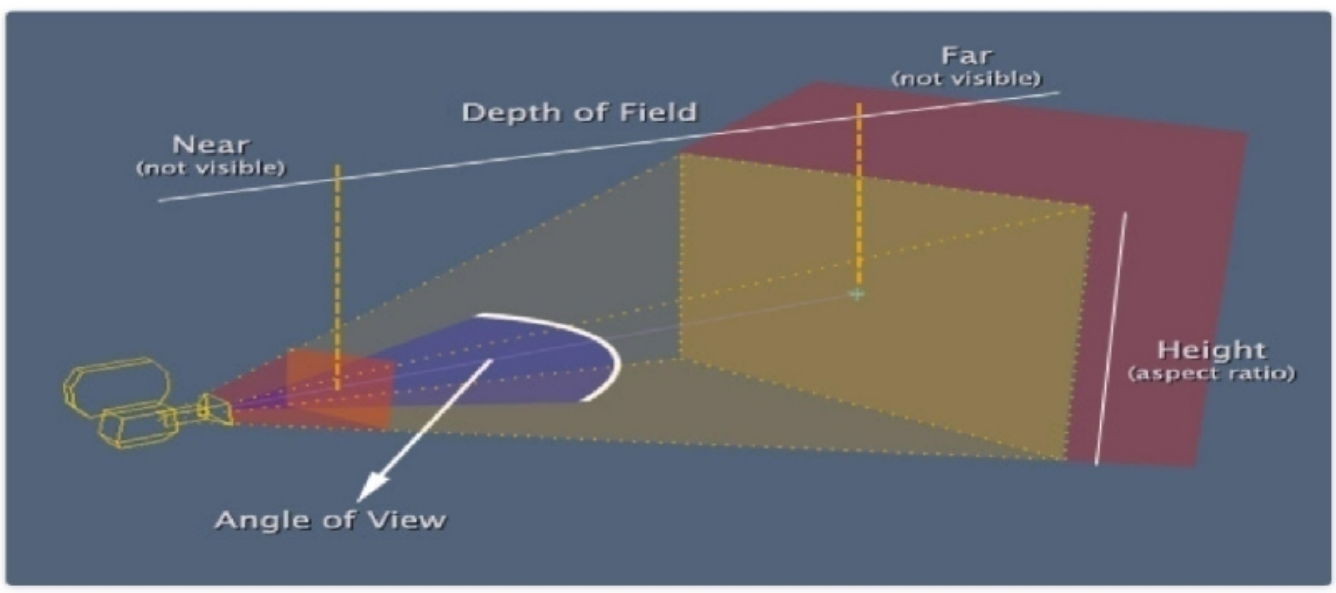

Figure 1: DOF and FOV of a compact camera.

$$
\begin{aligned}
& \text { DOF }=\frac{2 \mathrm{f}^{2} \mathrm{f}_{\mathrm{n}} \mathrm{c}(\mu+1)}{\mathrm{f}^{2} \mu^{2}-\mathrm{f}_{\mathrm{n}^{2}} \mathrm{c}^{2}} \\
& \text { FOV }=s \tan ^{-1}\left(\frac{\mathrm{d}(\mathrm{u}-\mathrm{f})}{2 \mathrm{uf}}\right)
\end{aligned}
$$

It is explicitly obvious from (1) and (2) that localisation and matching errors [8], are deeply engraved in camera measurements. It turns out that the data acquired with such cameras are difficult to work with especially in scene reconstruction and other certain critical application areas, and often does not give accurate result. It is important that these errors be adequately attended to and considerably reduced in order to improve accuracy, reliability, and computational performance issues in image processing applications such as $3 \mathrm{D}$ reconstruction. Hence, camera optical analysis has become an attractive research interest

\section{IMAGE FORMATION BASED ON WAVE OPTICS}

The energy that is captured by the camera is the visible part of electromagnetic (EM) spectrum, a self-propagating wave comprised of oscillating electric and magnetic fields generated by the acceleration of charged particles [10]. The propagation of EM radiation through inhomogeneous media with random fluctuations of local optical characteristics results in the formation of an optical wave that is characterised by random temporal and spatial distributions of its parameters such as intensity, phase, and, in general cases, its state of polarisation. Theoretical modelling of the propagation and the diffraction of electromagnetic wave at the camera aperture provides a vital input into the understanding of established imaging techniques and the development of new procedures.

Since the visible light shown in Figure 2 (a), reflected from scene objects, propagated and captured by the camera as depicted in Figure 2(b), [10], is an EM phenomenon of a particular wavelength, it can be described by Maxwell's equations. Maxwell's equations represent a unification of the works of Lorentz, Faraday, Ampere, and Gauss that predict the propagation of EM waves in free space at the speed of light $[11,12,13,14]$. Maxwell's equations which model EM waves are stated as: 
International Journal of Computer Science, Engineering and Applications (IJCSEA) Vol.4, No.6, December 2014

$\nabla \cdot \overrightarrow{\mathrm{E}}=\frac{\rho}{\varepsilon} \quad$ Gauss slaw

$\nabla \times \overrightarrow{\mathrm{E}}=\frac{-\partial \overrightarrow{\mathrm{B}}}{\partial \mathrm{t}} \quad$ Faraday' slaw

$\nabla \times \overrightarrow{\mathrm{H}}=\frac{\partial \overrightarrow{\mathrm{D}}}{\partial \mathrm{t}}+\overrightarrow{\mathrm{I}} \quad$ Ampere'slaw

$\nabla \cdot \overrightarrow{\mathrm{B}}=0 \quad$ Fluxlaw
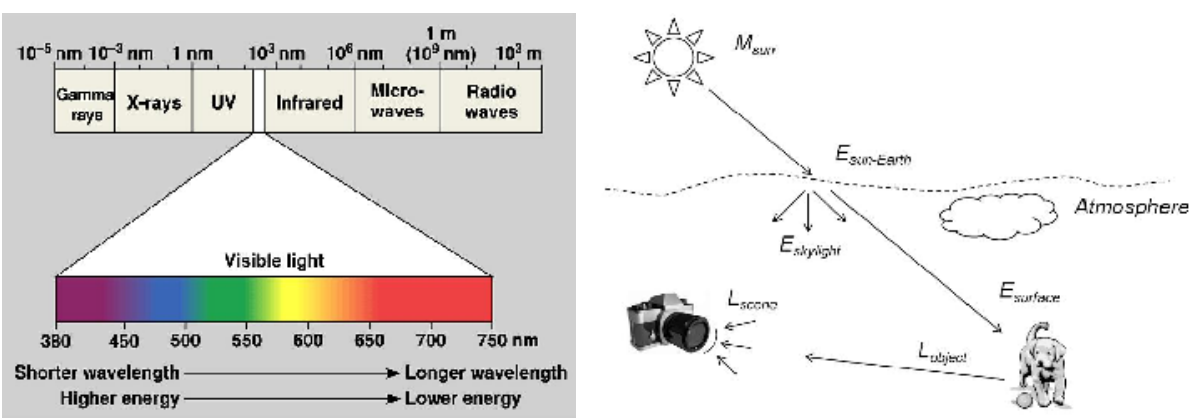

Figure 2: (a) Visible light spectrum, (b) the sun as a radiant source.

Where $\nabla$ is the del operator, $\overrightarrow{\mathrm{E}}$ and $\vec{B}$ are electric and magnetic respectively. $\overrightarrow{\mathrm{D}}$ is the electric displacement, $\overrightarrow{\mathrm{H}}$ defines the magnetic intensity, $\overrightarrow{\mathrm{J}}$ is the conduction current density in a volume, $\varepsilon$ is the permittivity, and $\rho$ is the charge density.

Equations (4) and (6) are valid only if position and time dependent magnetic $\vec{A}(\vec{r}, \mathrm{t})$ and electric $\varphi(\vec{r}, t)$ potentials exist at a field point on the camera sensor such that:

$$
\begin{aligned}
& \overrightarrow{\mathrm{B}}=\nabla \times \overrightarrow{\mathrm{A}} \\
& \overrightarrow{\mathrm{E}}=-\nabla \varphi-\frac{\partial \overrightarrow{\mathrm{A}}}{\partial \mathrm{t}}
\end{aligned}
$$

substituting $\overrightarrow{\mathrm{E}}$ from (8) in (3), we get

$$
\begin{aligned}
& \nabla \cdot\left(-\nabla \varphi-\frac{\partial \overrightarrow{\mathrm{A}}}{\partial \mathrm{t}}\right)=\frac{\rho}{\varepsilon} \\
& \frac{1}{\mathrm{c}^{2}} \frac{\partial^{2} \varphi}{\partial \mathrm{t}^{2}}-\nabla^{2} \varphi=\frac{\rho}{\varepsilon}
\end{aligned}
$$

using (7) in (4) we obtain

$$
\nabla \times \overrightarrow{\mathrm{E}}=\frac{-\partial(\nabla \times \overrightarrow{\mathrm{A}})}{\partial \mathrm{t}}=\frac{-\nabla \times \partial \overrightarrow{\mathrm{A}}}{\partial \mathrm{t}}
$$

rearranging (11) gives

$$
\nabla \times\left(\overrightarrow{\mathrm{E}}+\frac{\partial \overrightarrow{\mathrm{A}}}{\partial \mathrm{t}}\right)=0
$$

Two constitutive relationships can be written. These are 
International Journal of Computer Science, Engineering and Applications (IJCSEA) Vol.4, No.6, December 2014

$$
\begin{aligned}
& \overrightarrow{\mathrm{D}}=\varepsilon \overrightarrow{\mathrm{E}} \\
& \overrightarrow{\mathrm{B}}=\overrightarrow{\mathrm{H}}
\end{aligned}
$$

Equation (12) represents the infinitesimal rotation of the electric field. Since it is irrotational, it means it can be expressed as the gradient of electric potential $\varphi(\vec{r}, \mathrm{t})$. Hence we can write

$\vec{E}+\frac{\partial \vec{A}}{\partial t}=-\nabla \varphi$

Since the magnetic and electric potentials are not uniquely defined, there is the need to impose a constraint based on the gauge invariance of Maxwell's equations. The constrain widely applied is known as Lorenz condition and is written as

$$
\nabla \cdot \overrightarrow{\mathrm{A}}+\frac{1}{\mathrm{c}^{2}} \frac{\partial \varphi}{\partial \mathrm{t}}=0
$$

where $\mathrm{c}$ is the speed of light. $\mathrm{c}=\sqrt{\varepsilon \mu} . \varepsilon$ and are the dielectric parameters of the medium, i.e permittivity and permeability of space. Equation (5) can be rearrange as

$\nabla \times \overrightarrow{\mathrm{H}}-\frac{\partial \overrightarrow{\mathrm{D}}}{\partial \mathrm{t}}=\overrightarrow{\mathrm{J}}$

using (13), (14), and (16) in (17) we obtain

$$
\begin{aligned}
& \nabla \times(\nabla \times \overrightarrow{\mathrm{A}})-\frac{1}{\mathrm{c}^{2}} \frac{\partial}{\partial \mathrm{t}}\left(-\nabla \varphi-\frac{\partial \overrightarrow{\mathrm{A}}}{\partial \mathrm{t}}\right)=\overrightarrow{\mathrm{J}} V \\
& \frac{1}{\mathrm{c}^{2}} \frac{\partial^{2} \overrightarrow{\mathrm{A}}}{\partial \mathrm{t}^{2}}-\nabla^{2} \overrightarrow{\mathrm{A}}=\overrightarrow{\mathrm{J}}
\end{aligned}
$$

The expressions in (20) and (21) constitute the gauge invariance of Maxwell's equations which leaves $\vec{E}$ and $\vec{B}$ changed [15] for any scalar function $\mathrm{f}(\vec{r}, \mathrm{t})$.

$\varphi^{\prime}=\varphi-\frac{\partial f}{\partial t}$

$\overrightarrow{\mathrm{A}}=\overrightarrow{\mathrm{A}}+\nabla \mathrm{f}$

Applying (20) and (21) to (16), we can write

$\nabla \cdot \overrightarrow{\mathrm{A}^{\prime}}+\frac{1}{\mathrm{c}^{2}} \frac{\partial \varphi^{\prime}}{\partial \mathrm{t}}=\nabla \cdot \overrightarrow{\mathrm{A}}+\frac{1}{\mathrm{c}^{2}} \frac{\partial \varphi}{\partial \mathrm{t}}-\frac{1}{\mathrm{c}^{2}} \frac{\partial^{2} \mathrm{f}}{\partial \mathrm{t}^{2}}+\nabla^{2} \mathrm{f}$

Comparing (16) and (22), we have

$\frac{1}{c^{2}} \frac{\partial^{2} \mathrm{f}}{\partial \mathrm{t}^{2}}-\nabla^{2} \mathrm{f}=\nabla \cdot \overrightarrow{\mathrm{A}}+\frac{1}{\mathrm{c}^{2}} \frac{\partial \varphi}{\partial \mathrm{t}}$

Equation (23) is an inhomogeneous wave equation whose solution $\mathrm{f}$ could be used to refine $\overrightarrow{\mathrm{A}}$ and $\varphi$ so that equation (16) is satisfied.

Therefore, (10) and (19) are wave equations for the potentials which are representative of Maxwell's equations. The potentials can be computed if both conduction current density and charge density are known. In each of these two equations, the right hand side represents a continuous source function. In image acquisition using a camera, based on Huygens' principle, 
International Journal of Computer Science, Engineering and Applications (IJCSEA) Vol.4, No.6, December 2014 discrete functions are considered since any scene object is a set of point sources.

Consider a source point located on a scene object of volume $\mathrm{V}$ of known densities as shown in Figure 3. On the basis of the principle of causality, it takes $\frac{\mathrm{R}}{\mathrm{c}}$ seconds for the wave front emanating from the source $\vec{r}^{\prime}$ to reach any field points $\vec{r}$. Hence the potentials computed using (10) and (19) are referred to as retarded potentials.

Assuming a source point of arbitrary function $f(t),(10)$ will take the form

$$
\frac{1}{c^{2}} \frac{\partial^{2} \varphi}{\partial t^{2}}-\nabla^{2} \varphi=f(t) \delta^{(3)}(r)
$$

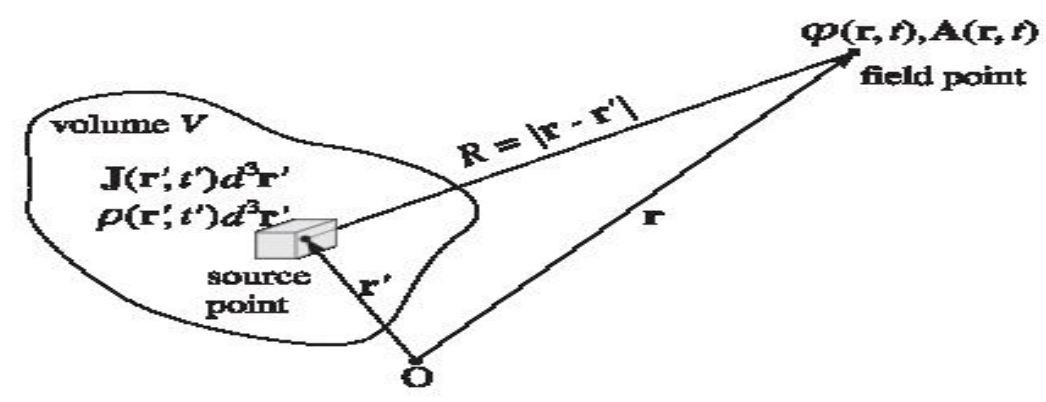

Figure 3: Retarded potentials generated by a localized current/charge distribution.

where $\delta^{(3)}(r)$ is a three-dimensional (3D) delta function. A delta function is defined only for $\mathrm{x}=0$. The value at $\mathrm{x}=0$ is infinity.

Also, assuming the solution of (24) at a retarded time $t^{\prime}=t-\frac{R}{c}$ is

$$
\varphi g(\vec{r}, t)=\frac{f\left(t^{\prime}\right)}{4 \pi r}=\frac{f\left(t-\frac{R}{c}\right)}{4 \pi r}
$$

Using a green's function $\mathrm{g}(\overrightarrow{\mathrm{r}})$ such that

$$
g(\vec{r})=\frac{1}{4 \pi r}
$$

Therefore, (25) becomes

$$
\begin{aligned}
& \varphi(\vec{r}, \mathrm{t})=\mathrm{f}\left(\mathrm{t}-\frac{\mathrm{r}}{\mathrm{c}}\right) \mathrm{g}(\overrightarrow{\mathrm{r}}) \\
& \text { from }(26) \text { we obtain } \\
& \nabla \mathrm{g}=-\hat{\mathrm{r}} \frac{\mathrm{g}}{\mathrm{r}} \\
& \nabla^{2} \mathrm{~g}=-\delta^{(3)}(\mathrm{r})
\end{aligned}
$$

$\hat{r}$ is a unit vector in the radial direction. The differential of the numerator of (25) with respect to $r$ is: 
Also

$\nabla \mathrm{f}=-\mathrm{r}_{\mathrm{c}}^{-}$

$\nabla^{2} f=\frac{1}{c^{2}} f-\frac{2}{c r}$

According to [15]

$$
\begin{aligned}
& \nabla^{2} \varphi=\nabla^{2}(f g)=2 \nabla f \cdot \nabla g+g \nabla^{2} f+f \nabla^{2} g \\
& \nabla^{2} \varphi=\frac{1}{c^{2}} f g-f\left(t-\frac{r}{c}\right) \delta^{(3)}(\vec{r})
\end{aligned}
$$

from (27)

$$
\frac{1}{c^{2}} \frac{\partial^{2} \varphi}{\partial t^{2}}=\frac{1}{c^{2}} f g
$$

Therefore, (34) becomes

$$
\frac{1}{c^{2}} \frac{\partial^{2} \varphi}{\partial t^{2}}-\nabla^{2} \varphi=f(t) \delta^{(3)}(\vec{r})
$$

This means that starting with the causal solution of (25) and the use of green's function for electrostatic Coulomb problem, it is possible to derive the corresponding wave equation (36) which is the same as (24). Therefore, the causal solution of (25) is correct.

For another source point at another location with the considered volume of Figure 3, $\vec{r}$ becomes $\vec{r}-\vec{r}$. As a result of this change, equation (36) becomes

$\frac{1}{c^{2}} \frac{\partial^{2} \varphi}{\partial t^{2}}-\nabla^{2} \varphi=f(t) \delta^{(3)}(\overrightarrow{\mathrm{r}}-\overrightarrow{\mathrm{r}})$

The causal solution of (37) therefore can be written as

$\varphi(\overrightarrow{\mathrm{r}}, \mathrm{t})=\frac{\mathrm{f}\left(\overrightarrow{\mathrm{r}},\left(\mathrm{t}-\frac{\mathrm{R}}{\mathrm{c}}\right)\right)}{4 \pi(\overrightarrow{\mathrm{r}}-\overrightarrow{\mathrm{r}})}$

Therefore, the total source function due to all the discrete source points in the considered volume can be obtained by performing volume integral. As such we have

$f(\vec{r}, t)=\iint f(\vec{r}, t) \delta^{(3)}(\vec{r}-\overrightarrow{\vec{r}}) d x d y d z \vec{r}$

The potential $\varphi(\overrightarrow{\mathrm{r}}, \mathrm{t})$ corresponding to the source expressed in (39) is obtained as

$\varphi(\overrightarrow{\mathrm{r}}, \mathrm{t})=\iint \frac{f\left(\overrightarrow{\mathrm{r}},\left(\mathrm{t}-\frac{\mathrm{R}}{\mathrm{c}}\right)\right)}{4 \pi(\overrightarrow{\mathrm{r}}-\overrightarrow{\mathrm{r}})} \mathrm{dx} d y d z \overrightarrow{\mathrm{r}}$

In conclusion, (40) is the causal solution to the general wave equation expressed in (34). Using a similar argument, $\vec{A}(\vec{r}, t)$ can be written as 
International Journal of Computer Science, Engineering and Applications (IJCSEA) Vol.4, No.6, December 2014

$\overrightarrow{\mathrm{A}}(\overrightarrow{\mathrm{r}}, \mathrm{t})=\iint \frac{f\left(\overrightarrow{\mathrm{r}},\left(\mathrm{t}-\frac{\mathrm{R}}{\mathrm{C}}\right)\right)}{4 \pi(\overrightarrow{\mathrm{r}}-\overrightarrow{\mathrm{r}})} \mathrm{d} x \mathrm{dydz \vec { \textrm {r } }}$

This idea can be applied in order to compute the retarded potentials to the wave equations (10) and (19) where $f(\vec{r}, \mathrm{t})=\frac{\rho(\vec{r}, t)}{\varepsilon}, f(\vec{r}, t)=\vec{J}(\vec{r}, t)$, and $R=|\vec{r}-\vec{r}|$. i.e

$\varphi(\overrightarrow{\mathrm{r}}, \mathrm{t})=\iint \frac{\rho(\overrightarrow{\mathrm{r}})}{4 \pi \varepsilon \mathrm{R}} \mathrm{dxdydz \vec { \textrm {r } }}$

$\overrightarrow{\mathrm{A}}(\overrightarrow{\mathrm{r}}, \mathrm{t})=\iiint_{4 \pi \mathrm{R}}^{\overrightarrow{\mathrm{J}}(\overrightarrow{\mathrm{r}})} \mathrm{dxdydz} \overrightarrow{\mathrm{r}}$

In practice, the both conduction current density $\vec{J}$ and electric charge density $\rho$ depend on the object to be capture by the camera.

If the source point oscillates with respect to time, then $\varphi(\vec{r}, \mathrm{t})=\varphi(\vec{r}) \mathrm{e}^{\mathrm{j} \omega \mathrm{t}}, \overrightarrow{\mathrm{A}} \mathrm{A}(\overrightarrow{\mathrm{r}} \mathrm{r}, \mathrm{t})=\overrightarrow{\mathrm{A}}(\overrightarrow{\mathrm{r}}) \mathrm{e}^{\mathrm{j} \omega \mathrm{t}}$, $\rho(\vec{r}, t)=\rho(\vec{r}) e^{j \omega t}, \vec{J}(\vec{r}, t)=\vec{J}(\vec{r}) e^{j \omega t}$. Where $\omega$ is the angular frequency of the oscillation, $j$ is the complex component. Therefore, the retarded potentials will take to different forms as

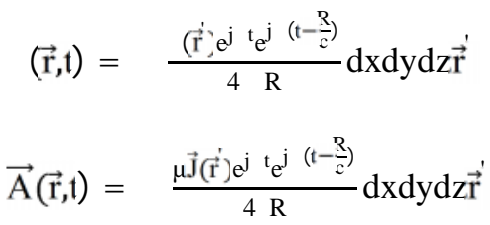

The simplification of (44) and (45) yield

$\varphi(\overrightarrow{\mathrm{r}}, \mathrm{t})=\iint \frac{\rho(\overrightarrow{\mathrm{r}}) \mathrm{e}^{-j \mathrm{k} R}}{4 \pi \varepsilon \mathrm{R}} \mathrm{dxdydz \vec { \textrm {r } }}$

$\overrightarrow{\mathrm{A}}(\overrightarrow{\mathrm{r}}, \mathrm{t})=\iiint_{\frac{\vec{J}(\overrightarrow{\mathrm{r}}) \mathrm{e}^{-j \mathrm{k} R}}{4 \pi \mathrm{R}}}^{\mathrm{d}} \mathrm{xdydz \vec { \textrm {r } }}$

where $\mathrm{k}=\frac{\omega}{\mathrm{c}}$ is referred to as the wave number.

Therefore, the magnetic and electric fields of (7) and (8) can be calculated if $\vec{A}(\vec{r}, \mathrm{t})$ and $\varphi(\overrightarrow{\mathrm{r}}, \mathrm{t})$ are known. However, (8) can be made to depend only on vector potential $\vec{A}(\vec{r}, \mathrm{t})$ with further simplification. Re-writing Lonrenz condition and recall that $c=\sqrt{\varepsilon \mu}$, and $\frac{\partial}{\partial t}=j \omega$, we can have

$$
\nabla \cdot \vec{A}+j \omega \varepsilon \varphi=0
$$

making $\varphi$ the subject of the formula, we get Type equation here.

$$
\varphi=\frac{-1}{j \omega \varepsilon} \nabla \cdot \overrightarrow{\mathrm{A}}
$$

Therefore, the electric field expressed in (8) becomes 


$$
\left\{\begin{array}{c}
\vec{E}=\frac{1}{j \omega \varepsilon}\left(\nabla(\nabla \cdot \vec{A})+k^{2} \vec{A}\right) \\
\vec{E}=\frac{1}{j \omega \varepsilon}(\nabla \times(\nabla \times \vec{A})-\vec{J})
\end{array}\right\}
$$

In electromagnetic imaging problems, interest focuses on the behaviour of the scattered EM wavefield generated by variations in the material parameters $\varepsilon$, and $\sigma . \sigma$ is the conductivity of the medium. Also

$\overrightarrow{\mathrm{H}}=\frac{1}{-} \nabla \times \overrightarrow{\mathrm{A}}$

In optical imaging, the camera sensor is considered to be located in the fields that have radiated away from their current sources (scene objects). This is because they carry large power. Such farfields have to satisfy the condition $\overrightarrow{\mathrm{r}} \gg \overrightarrow{\mathrm{r}}$ or $\vec{r}>1$. Where 1 is the extent of the current distribution in the source. The situation usually obtained in practice and depicted in Figure 4 is that at far distances the sides PP' and PQ of the triangle PQP' are almost equal.

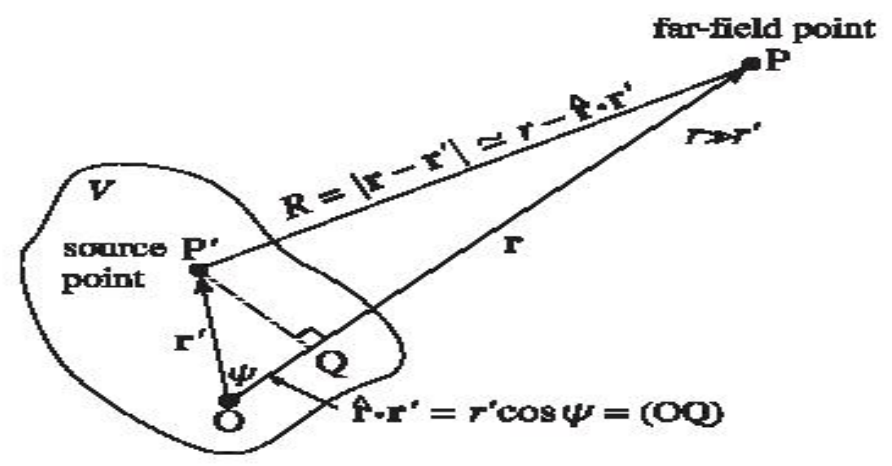

Figure 4: Far-field approximation

Applying cosine rule to triangle $\mathrm{PQP}^{\prime}$ we get

$$
R=|\vec{r}-\vec{r}|=\frac{\left(r^{2}+r^{2}-2 r r^{\prime} \cos \varphi\right)^{1}}{2}
$$

when $r$ is factored out of the square root, we can write

$$
\mathrm{R}=\mathrm{r} \frac{\left(1-2 \frac{\mathrm{r}_{\mathrm{r}}^{\prime}}{\mathrm{r}} \cos \psi+\frac{r^{2}}{\mathrm{r}^{2}}\right)^{1}}{2}
$$

Considering the fact that $\overrightarrow{\mathrm{r}} \gg \overrightarrow{\mathrm{r}}$ and applying Binomial approximation to (53), we have the reduced form as

$\mathrm{R}=\mathrm{r}\left(1-\frac{1}{2}\left(2 \frac{\mathrm{r}}{\mathrm{r}} \cos \psi\right)\right)$

with further simplification, and recognizing that the dot product $\vec{M} \cdot \vec{N}$ of two vectors $\vec{M}$ and $\vec{N}$ that are positioned with respect to each other at an angle $\theta$ between them is $|\mathrm{M}||\mathrm{N}| \cos \theta$, we get

$\mathrm{R}=\mathrm{r}-\mathrm{r}^{\prime} \cos \psi=\mathrm{r}-\hat{\mathrm{r}}^{\prime} \mathrm{r}^{\prime} \simeq \mathrm{r}$ 
We now substitute the approximate form of $\mathrm{R}$ (the distance from a volume element $d V$ to the point of observation) in the denominator part of (47). The approximation allows for component terms that constitute the waves that are propagated towards the camera sensor. Hence we obtain

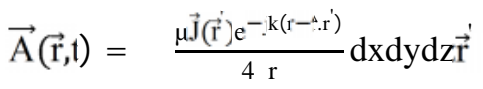

rearranging (56) we get

$\overrightarrow{\mathrm{A}}(\overrightarrow{\mathrm{r}}, \mathrm{t})=\frac{\mu \mathrm{e}^{-\mathrm{jkr}}}{4 \pi \mathrm{r}} \iiint \overrightarrow{\mathrm{J}}\left(\overrightarrow{\mathrm{r}}^{\prime}\right) \mathrm{e}^{\mathrm{jkr} \cdot \mathrm{r}^{\prime}} \mathrm{dxdydz \vec { \textrm {r } } ^ { \prime }}$

To use the camera aperture as field source of the radiated fields, a variation of the HuygensFresnel principle needs to be used. This principle states that the points on each wavefront become the sources of secondary spherical waves propagating outwards and whose superposition generates the next wavefront. Therefore, rectangular aperture will be considered.

\subsection{Rectangular Aperture}

In $[16,17,18]$, it is thought that an aperture can be a source. We are now interested in the field distribution emanating from it. If we consider an infinitesimally small camera aperture (pinhole) as shown in Figure 5 to be a source of surface $d S$, with the surface current density distribution $\mathrm{J}_{\mathrm{s}}$, then (57) can be written as

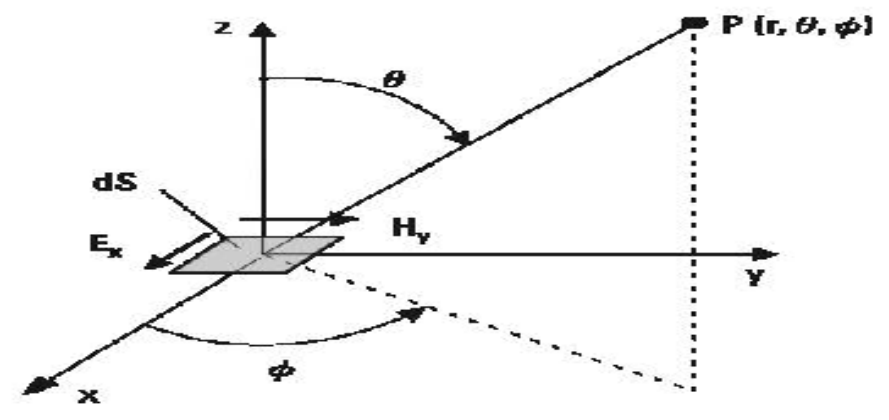

Figure 5: Radiating surface element

$$
\mathrm{d} \overrightarrow{\mathrm{A}}(\overrightarrow{\mathrm{r}}, \mathrm{t})=\frac{\mathrm{e}^{-\mathrm{jkr}}}{4 \pi \mathrm{r}} \cdot \vec{J} \mathrm{~J}_{\mathrm{s}}(\overrightarrow{\overrightarrow{\mathrm{r}}}) \mathrm{e}^{\mathrm{j} \mathrm{k} \hat{\mathrm{r}} \cdot \mathrm{r}} \mathrm{d} \mathrm{S} \overrightarrow{\mathrm{r}}
$$

$\overrightarrow{\mathrm{J}_{\mathrm{s}}}$ is expressed as

$\mathrm{J}_{\mathrm{s}}=\hat{\mathrm{n}} \times \overrightarrow{\mathrm{H}_{\mathrm{y}}}=\frac{-\mathrm{E}_{\mathrm{x}}}{\eta} \mathrm{r}^{\prime}$

where $\eta$ is the wave impedance. $E_{x}$ and $H_{y}$ are the electric and magnetic fields in the $x$ and $y$ directions.

The wave exiting the aperture is spherical. In the region farther from the aperture, the field distribution can be approximated as parabolic wave. This is referred to as the Fresnel region. As the waves travel even further away from the aperture the spherical waves become approximately 
plane waves. Usually the camera lens will bring the propagating light in the far-field (Fraunhofer diffraction region) to a focus on the sensor to form the image.

To model the far-field, the solutions of Maxwell's equations and the directional patterns of a source are best described in spherical coordinates since the fields radiated by sources of finite dimensions are spherical. Therefore, the magnetic vector potential is expressed in component form as

$$
\begin{aligned}
\mathrm{A}_{\mathrm{r}} & =\sin \theta \cos \phi \mathrm{A}_{\mathrm{x}} \\
\left\{\mathrm{A}_{\theta}\right. & \left.=\cos \theta \cos \phi \mathrm{A}_{\mathrm{x}}\right\} \\
\mathrm{A}_{\phi} & =-\sin \phi \mathrm{A}_{\mathrm{x}} \\
\nabla \times \overrightarrow{\mathrm{A}} & =\hat{\mathrm{r}} \frac{1}{\mathrm{r} \sin \theta}\left(\frac{\partial\left(\sin \theta \mathrm{A}_{\phi}\right)}{\partial \theta}-\frac{\partial \mathrm{A}_{\theta}}{\partial \phi}\right)+\hat{\theta} \frac{1}{\mathrm{r}}\left(\frac{1}{\sin \theta} \frac{\partial \mathrm{A}_{\mathrm{r}}}{\partial \phi}-\frac{\partial\left(\mathrm{rA}_{\phi}\right)}{\partial \mathrm{r}}\right)+\hat{\phi} \frac{1}{\mathrm{r}}\left(\frac{\partial(\mathrm{rA} \theta}{\partial \mathrm{r}}-\frac{\partial \mathrm{A}_{\mathrm{r}}}{\partial \mathrm{r}}\right) \\
\nabla \times \overrightarrow{\mathrm{A}} & =\frac{-\partial \mathrm{A}_{\phi}}{\partial \mathrm{r}} \hat{\theta}+\frac{\partial \mathrm{A}_{\theta}}{\partial \mathrm{r}}
\end{aligned}
$$

Using (50) under the assumption of free-space (points removed from source, $\hat{J}=0$ ), [19] the electric field components in the $\theta$ and $\phi$ directions can be obtained as

$$
\begin{aligned}
& \mathrm{E}_{\theta}=\frac{j \mathrm{E}_{\mathrm{x}} \mathrm{e}^{-\mathrm{jkr}}}{2 \lambda \mathrm{r}}(\cos \theta+1) \cos \phi \mathrm{d} S \\
& \mathrm{E}_{\theta}=-\sin \phi \frac{j \mathrm{E}_{\mathrm{x}} \mathrm{e}^{-\mathrm{k} \mathrm{kr}}}{2 \lambda \mathrm{r}}(\cos \theta+1) \mathrm{dS} \\
& \mathrm{H}_{\theta}=\frac{-\mathrm{E}_{\phi}}{\eta} \mathrm{H}_{\phi}=\frac{\mathrm{E}_{\theta}}{\eta} \mathrm{H}_{\mathrm{r}}=0 \mathrm{E}_{\mathrm{r}}=0
\end{aligned}
$$

For a camera aperture of dimensions a and b in the x-y plane shown in Figure 6, the radiated electric is the summation of all the contributions by the infinitesimally small sources within the dimension of the aperture. Therefore we can write

$$
E_{\theta}=\frac{j E_{x} e^{-j k r}}{2 \lambda r}(\cos \theta+1){ }_{-m}^{m}{ }_{-n}^{\eta} e^{-j k r . r} d x^{\prime} d y^{\prime}
$$

$$
\begin{aligned}
& \text { where } \mathrm{m}=\frac{\mathrm{b}}{2}, \mathrm{n}=\frac{\mathrm{a}}{2} \text {, and } \\
& \text { 个..r' }=(\cos \phi \sin \theta) \mathrm{x}^{\prime}+(\sin \phi \sin \theta) \mathrm{y}^{\prime}
\end{aligned}
$$




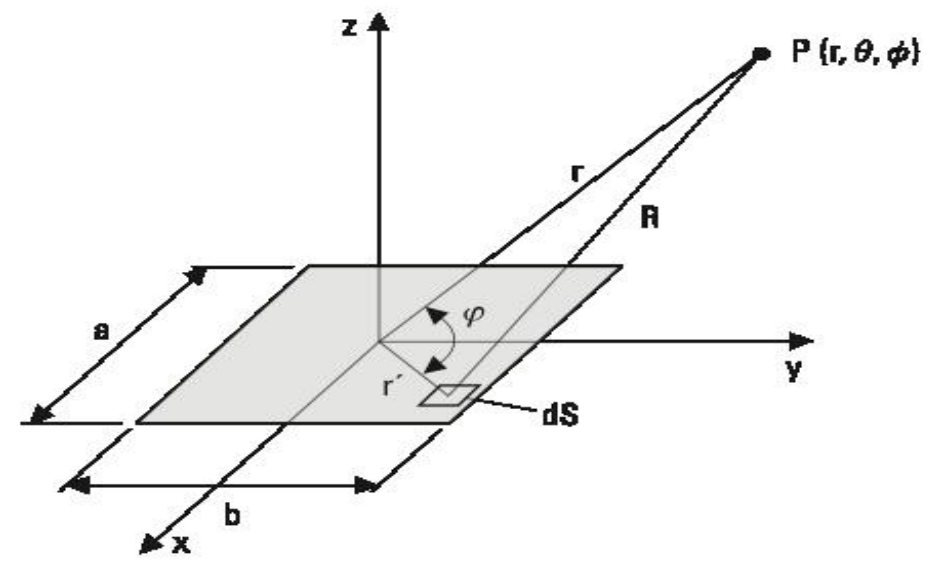

Figure 6: Radiation aperture of a by $\mathrm{b}$ dimension.

Therefore, (66) becomes

$$
\mathrm{E}_{\theta}=\left(\frac{\sin \left(\frac{1}{2} \mathrm{kbsin} \theta \sin \phi\right)}{\frac{1}{2} \mathrm{kbsin} \theta \sin \phi}\right)\left(\frac{\sin \left(\frac{1}{2} \mathrm{kasin} \theta \cos \phi\right)}{\frac{1}{2} \mathrm{kasin} \theta \cos \phi}\right) \sin \phi(1+\cos \theta) \frac{\mathrm{jab} \mathrm{E}_{\mathrm{x}}}{2 \lambda \mathrm{r}} \mathrm{e}^{-\mathrm{jkr}}
$$

where $\lambda$ is the wavelength. Similarly

$$
\mathrm{E}_{\phi}=\left(\frac{\sin \left(\frac{1}{2} \mathrm{kbsin} \theta \sin \phi\right)}{\frac{1}{2} \mathrm{kbsin} \theta \sin \phi}\right)\left(\frac{\sin \left(\frac{1}{2} \mathrm{kasin} \theta \cos \phi\right)}{\frac{1}{2} \mathrm{kasin} \theta \cos \phi}\right) \cos \phi(1+\cos \theta) \frac{\mathrm{jabE}}{2 \lambda \mathrm{r}} \mathrm{e}^{-\mathrm{jkr}}
$$

According to [16], $\mathrm{E}_{\theta}$ and $\mathrm{E}_{\phi}$ only exist in the yz-plane $\left(\phi=\frac{\pi}{2}\right)$ and xz-plane $(\phi=0)$ respectively. It is further stated that significant field is generated at small angles only if the aperture is large such that $\mathrm{a}$ and $\mathrm{b} \gg \lambda$. This means $\cos \theta \approx 1$ and $\sin \theta=\theta$. Therefore, the field pattern yz-plane ( $\mathrm{E}-$ plane) becomes

$\mathrm{E}_{\mathrm{r}}=\mathrm{E}_{\phi}=0, \mathrm{E}_{\theta}=\left(\frac{\sin \left(\frac{1}{2} \mathrm{~kb} \theta\right)}{\frac{1}{2} \mathrm{~kb} \theta}\right) \frac{\mathrm{jab}_{\mathrm{x}}}{\lambda \mathrm{r}} \mathrm{e}^{-\mathrm{jkr}}$

for the xz-plane, i.e ( $\mathrm{H}$ - plane), we have

$E_{r}=E_{\theta}=0, E_{\phi}=\left(\frac{\sin \left(\frac{1}{2} k a \theta\right)}{\frac{1}{2} k a \theta}\right) \frac{j a b E_{x}}{\lambda r} e^{-j k r}$

Equation (70) and (71) describe the 3D electric field distribution that is focused on the camera sensor.

Therefore, the intensity distribution $\mathrm{I}(\mathrm{x}, \mathrm{y})$ recorded on the camera sensor is

$\mathrm{I}(\mathrm{x}, \mathrm{y})=\left|\mathrm{E}_{\theta}\right|^{2}=\frac{\mathrm{E}_{\mathrm{x}^{2}}}{(\lambda \mathrm{r})^{2}}\left|\mathrm{ab}\left(\left(\frac{\sin \left(\frac{1}{2} \mathrm{ka} \theta\right)}{\frac{1}{2} \mathrm{ka} \theta}\right)^{2}+\left(\frac{\sin \left(\frac{1}{2} \mathrm{~kb} \theta\right)}{\frac{1}{2} \mathrm{~kb} \theta}\right)^{2}\right)\right|^{2}$

Equation (72) expresses the diffraction ability of the aperture. the consequence of this is that different scene points are made to spread out.

Experiment has shown that because camera sensors are not perfect, a scene point will always be 
blurred as shown in Figure 7. If a lens produces the same blurring effect irrespective of the position of the scene point, then the lens is said to be linear shift-invariant. Therefore, the blur point can be considered as the point PSF of the lens. The challenge now is how to obtain this PSF.

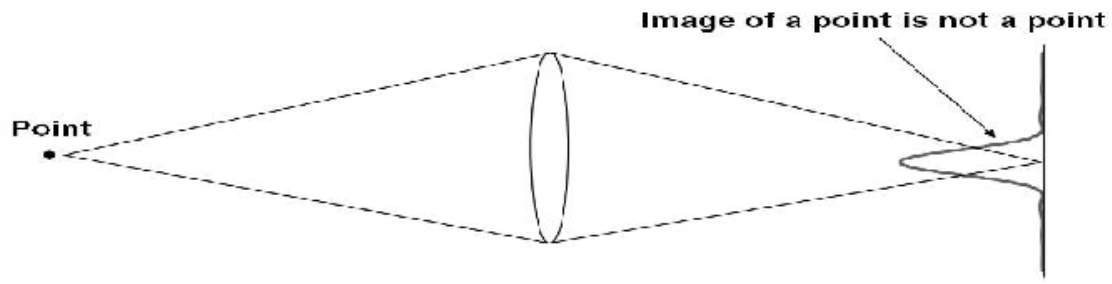

Figure 7: The image of a scene point is not a perfect point on the camera sensor.

From (3.70), it is observed that the $\mathrm{I}(\mathrm{x}, \mathrm{y})$ is directly proportional to PSF. We extract PSF as

$$
\mathrm{PSF}=\left.\mathrm{abl}\left(\left(\frac{\sin \left(\frac{1}{2} \mathrm{ka} \theta\right)}{\frac{1}{2} \mathrm{ka} \theta}\right)^{2}+\left(\frac{\sin \left(\frac{1}{2} \mathrm{~kb} \theta\right)}{\frac{1}{2} \mathrm{~kb} \theta}\right)^{2}\right)\right|^{2}
$$

Consider a scene as a set of points with different intensity value represented by $p(x, y)$. If the image of $\mathrm{p}(\mathrm{x}, \mathrm{y})$ is $\mathrm{P}(\mathrm{x}, \mathrm{y})$, then the image formation process can be expressed in convolution form as

$\mathrm{P}(\mathrm{x}, \mathrm{y})=\operatorname{PSF}(\mathrm{x}, \mathrm{y}) * \mathrm{p}(\mathrm{x}, \mathrm{y})$

Writing (74) in frequency domain we have

Fouriertransform $[\mathrm{P}(\mathrm{x}, \mathrm{y})]=$ Fouriertransform $[\mathrm{PSF}(\mathrm{x}, \mathrm{y}) * \mathrm{p}(\mathrm{x}, \mathrm{y})]$

Hence the Fourier transform of PSF is the optical transfer function (OTF) of the camera. It is expressed as

$\mathrm{OTF}=$ Fouriertransform $[\mathrm{PSF}(\mathrm{x}, \mathrm{y})]$

In the normalized form, the OTF is referred to as modulation transfer function (MTF). This is an important parameter used for performance characterization of cameras and images as shown in Figure 8(a).

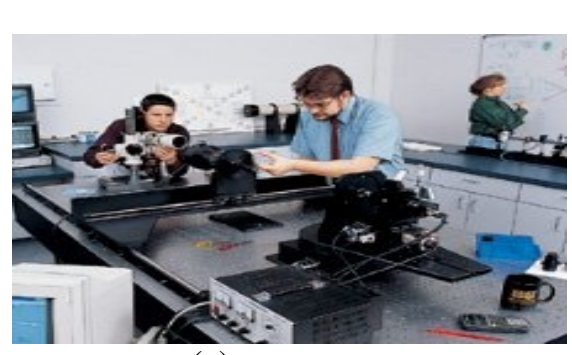

(a)

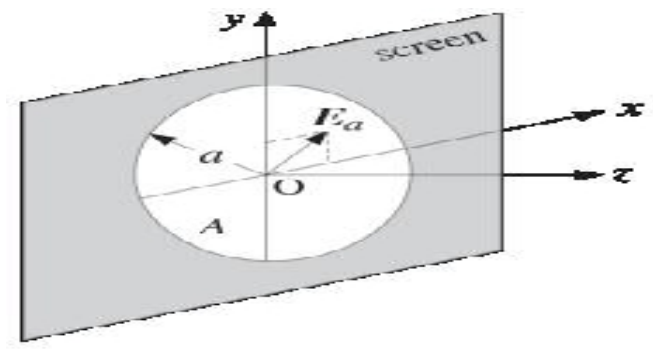

(b)

Figure 8: (a) Testing of cameras: http://www.edmundoptics.com/technical-resources-center/, (b) circular aperture. 


\section{SIMULATION RESULTS AND DISCUSSION}

A 3D plot of the field patterns can be seen in Figure 9. It can be observed that because the dimensions of the aperture are one wavelength, there are no sidelobes. However, when the aperture dimensions are a multiple of one wavelength, multiple sidelobes begin to appear. The reason for this observation can be found in (70) and (71). The E-plane field distribution is a function of the dimension $b$ of the aperture while for the H-plane it is dependent on a . Therefore, the number of sidelobes increases as the aperture dimension increases [19].

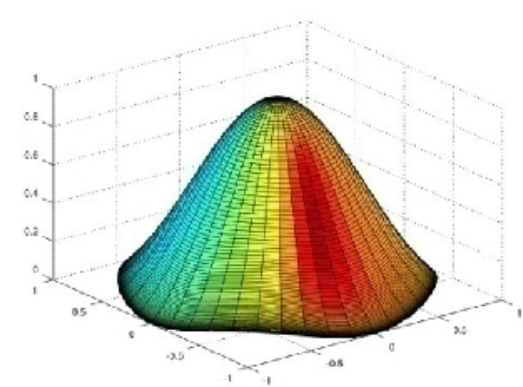

(a) $a=b=\lambda$

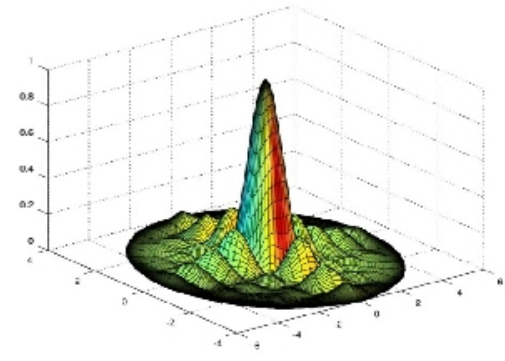

(c) $a=5 \lambda, b=4 \lambda$

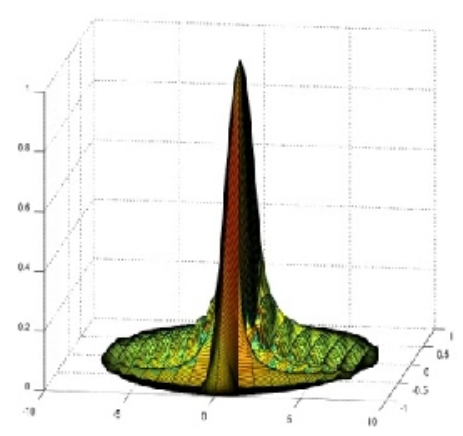

(e) $\mathrm{a}=8 \lambda, \mathrm{b}=\lambda$

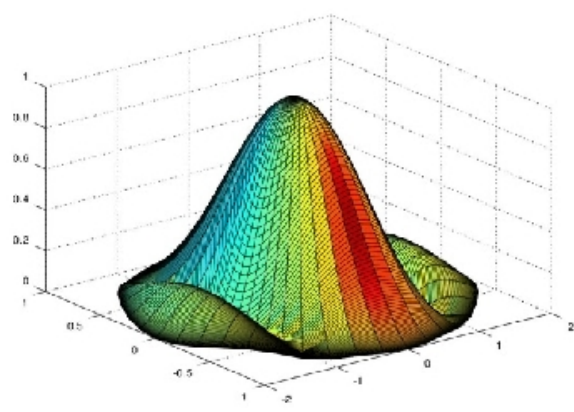

(b) $a=2 \lambda, b=\lambda$

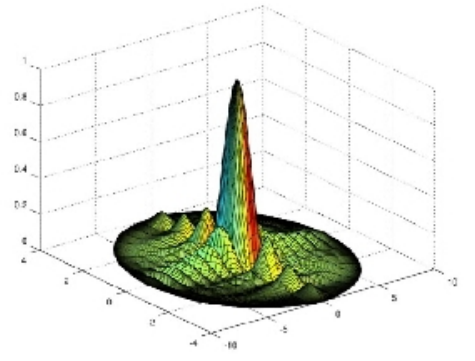

(d) $\quad \mathrm{a}=8 \lambda, \mathrm{b}=4 \lambda$

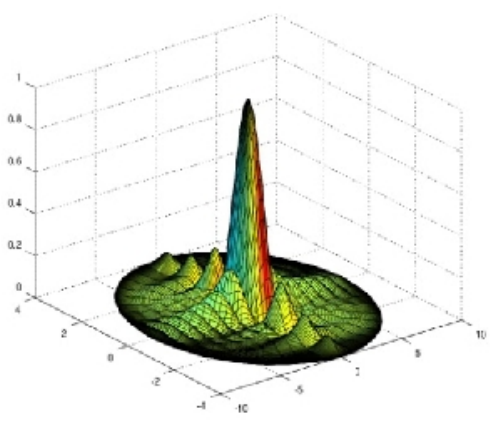

(f) $\mathrm{a}=7 \lambda, \mathrm{b}=2.5 \lambda$

Figure 9: 3D plot of radiation pattern focused on camera sensor based on the use of rectangular aperture. 
International Journal of Computer Science, Engineering and Applications (IJCSEA) Vol.4, No.6, December 2014

Also a plot of the field strength against theta $\theta$, for some aperture dimensions is give in Figure 10 and 11 .
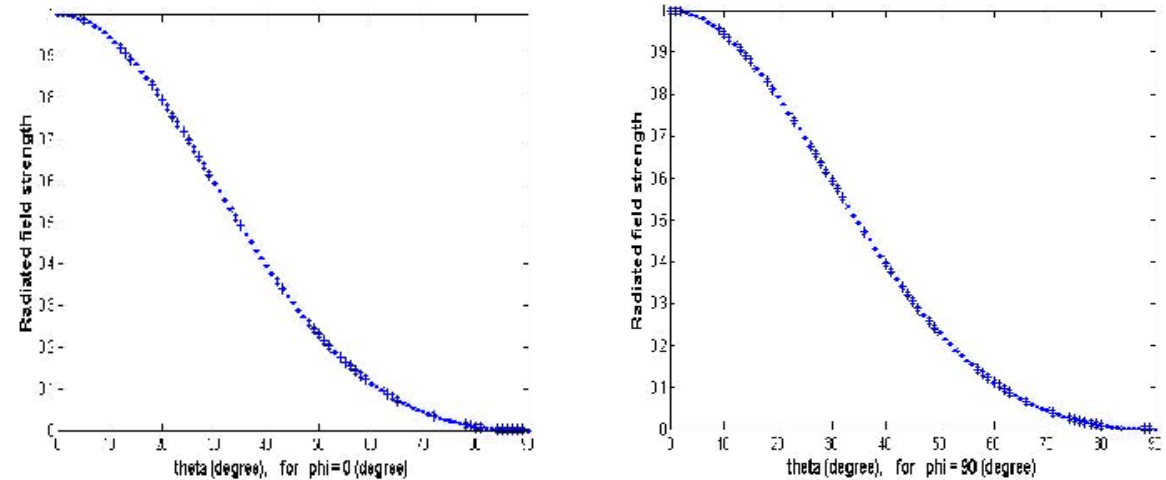

(a) $a=b=\lambda$ for $\phi=0^{\circ}$

(b) $a=b=\lambda$ for $\phi=90^{\circ}$
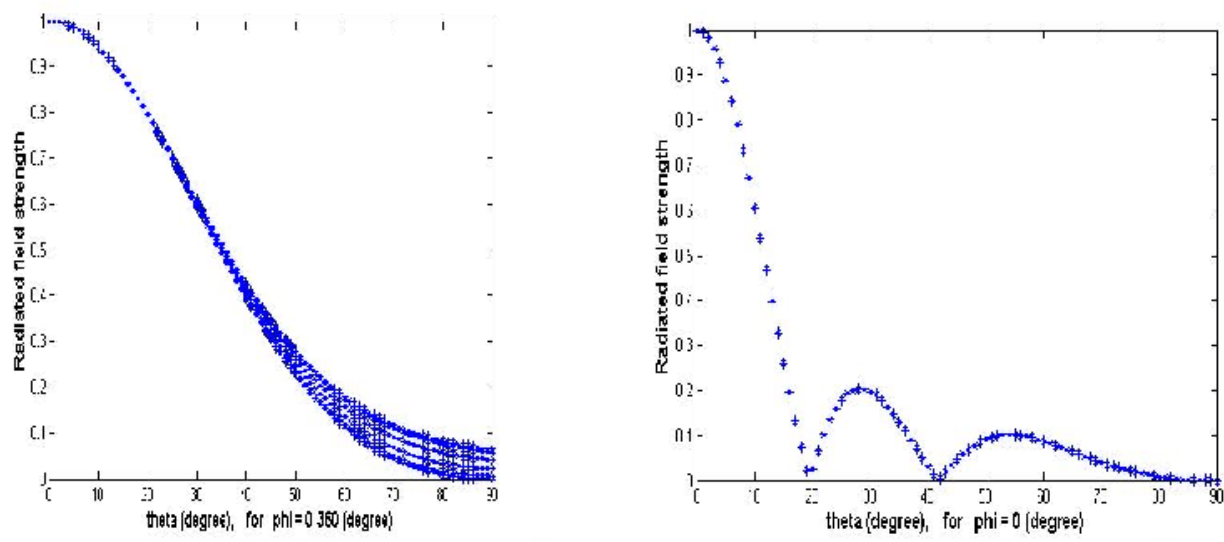

(c) $a=b=\lambda$ for $\phi=0: 360^{\circ}$

(d) $a=3 \lambda, b=\lambda$ for $\phi=0^{\circ}$
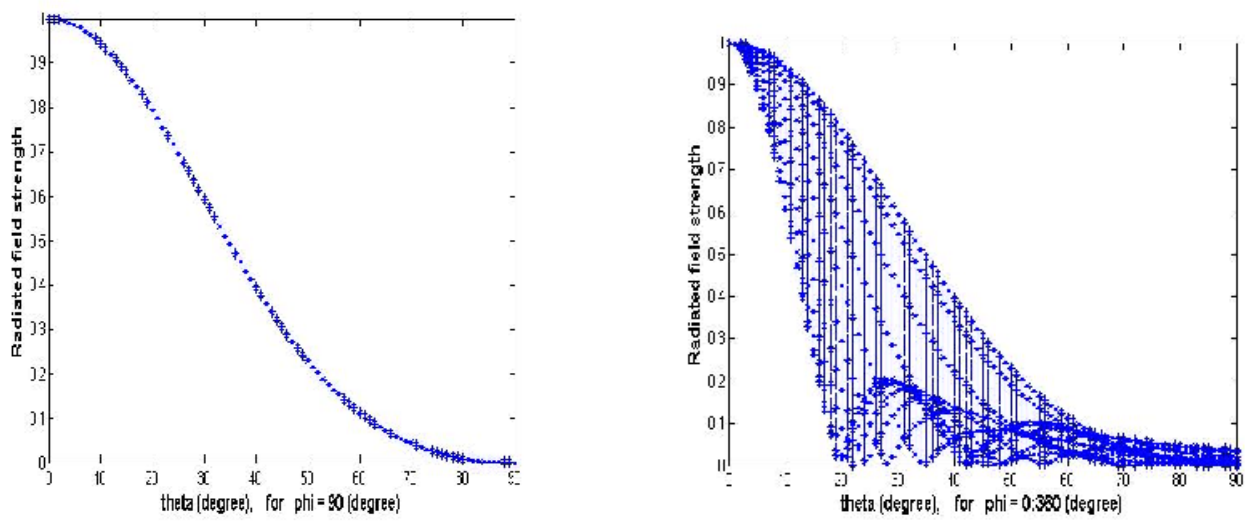

(e) $a=3 \lambda, b=\lambda$ for $\phi=90^{\circ}$

(f) $a=3 \lambda, b=\lambda$ for $\phi=0: 360^{\circ}$

Figure 10: 2D plot of field strength against theta. 
International Journal of Computer Science, Engineering and Applications (IJCSEA) Vol.4, No.6, December 2014
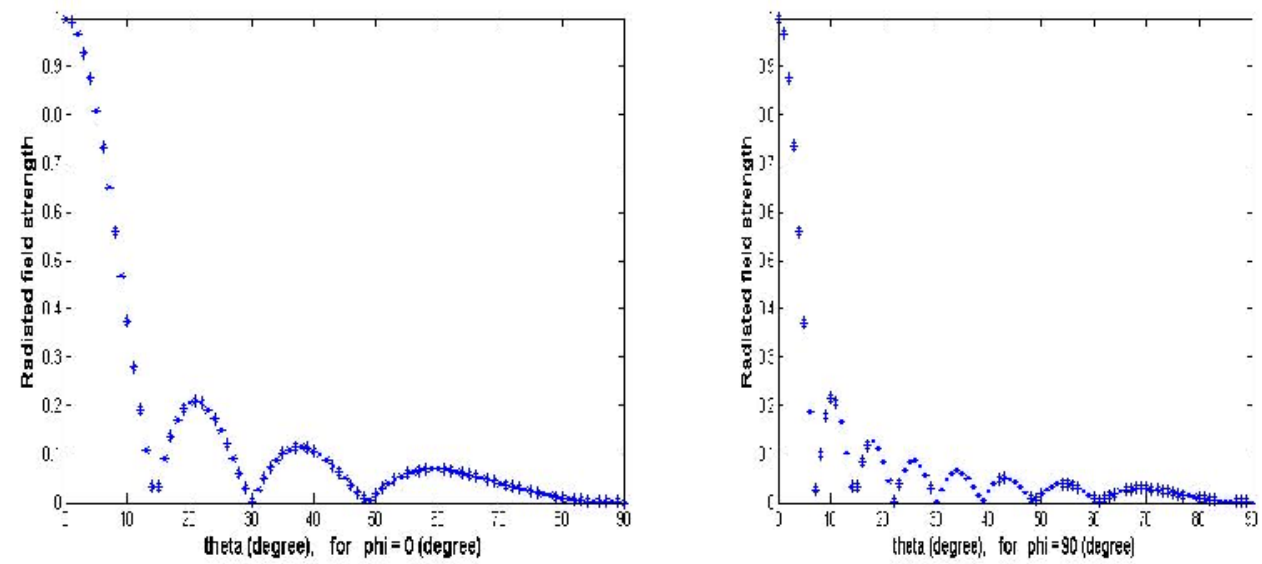

(a) $a=4 \lambda_{\ominus} b=8 \lambda$ for $\phi=0^{\circ}$

(b) $a=4 \lambda, b=8 \lambda$ for $\phi=90^{\circ}$
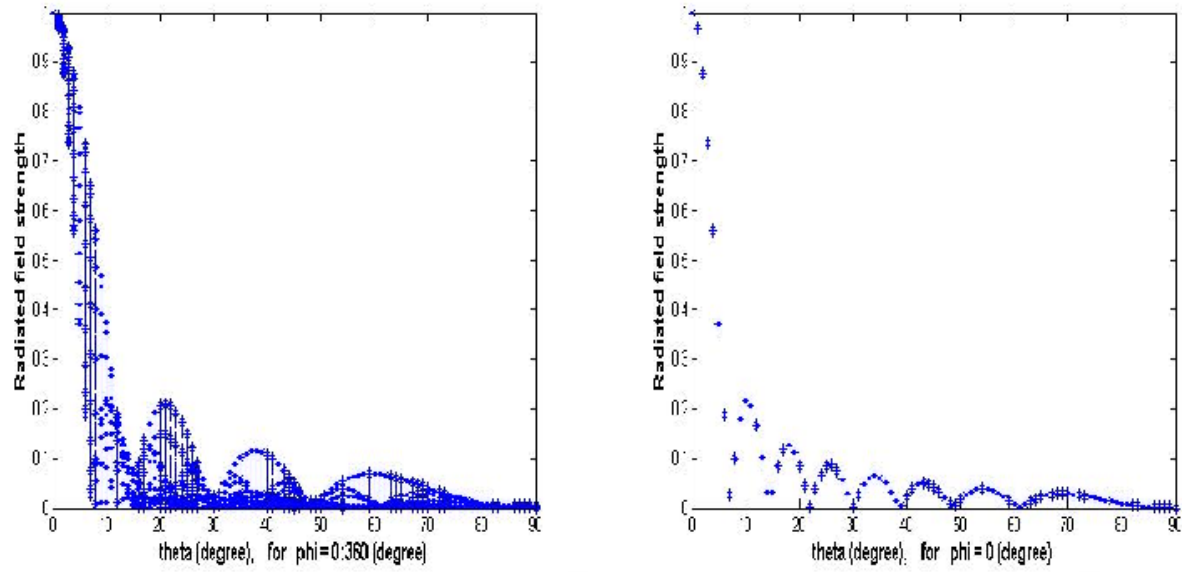

(c) $a=4 \lambda, b=8 \lambda$ for $\phi=0: 360^{\circ}$

(d) $a=8 \lambda, b=\lambda$ for $\phi=0^{\circ}$
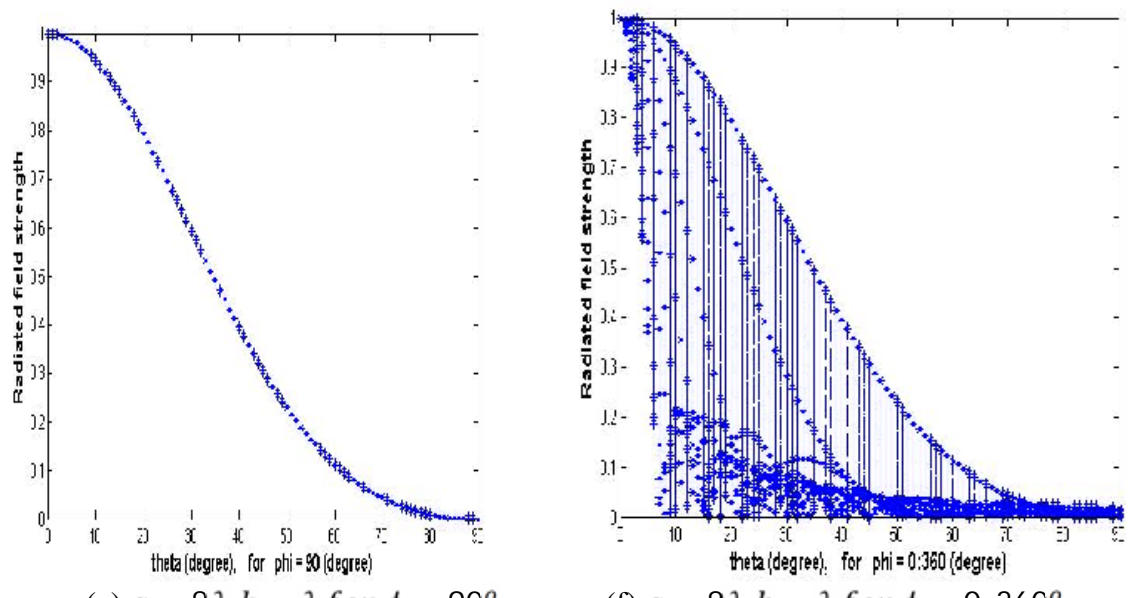

(e) $a=8 \lambda, b=\lambda$ for $\phi=90^{\circ}$

(f) $a=8 \lambda, b=\lambda$ for $\phi=0: 360^{\circ}$

Figure 11: 2D plot of field strength against theta. 


\section{CONCLUSION}

An important camera and image quality parameter known as MTF is the basis of the modelling presented in this chapter. This has been achieved through wave optics consideration of camera image formation. It is experimentally observed that the number of sidelobes increases as the aperture dimension increases. The sidelobe is a measure of the spread out of an imaged point. Therefore, a high quality image of a point would be produced when the camera aperture is one wavelength. A measure of the ability, in frequency domain, of a camera to produce an exact replica of a point is referred to as MTF. Consequently, this reflects in the overall quality of the image of an object which is usually a set of points. Also, MTF is use for objective quality assessment of both cameras and images.

\section{REFERENCES}

[1] K. Iizuka, Engineering optics, vol. 35. Springer, 2008.

[2] "Digital imaging," International Journal of Computer Assisted Radiology and Surgery, vol. 3, no. S1, pp. 3-10, 2008.

[3] A. Foi, A. Foi, S. Alenius, S. Alenius, V. Katkovnik, V. Katkovnik, K. Egiazarian, and K. Egiazarian, "Noise measurement for raw-data of digital imaging sensors by automatic segmentation of nonuniform targets," IEEE Sensors Journal, vol. 7, no. 10, pp. 1456-1461, 2007.

[4] R. D. Fiete, "Image chain analysis for space imaging systems," Journal of Imaging Science and Technology, vol. 51, no. 2, pp. 103-109, 2007.

[5] S. K. Nayar, "Catadioptric omnidirectional camera," in Computer Vision and Pattern Recognition, 1997. Proceedings., 1997 IEEE Computer Society Conference on, pp. 482-488, IEEE, 1997.

[6] B. Micusik and T. Pajdla, Omnidirectional Camera Model and Epipolar Geometry Estimation by RANSAC with Bucketing?, pp. 83-90. Image Analysis, Springer, 2003.

[7] C.-K. Sung and C.-H. Lu, "Single-camera panoramic stereo system model based on skew ray tracing," Optik - International Journal for Light and Electron Optics, vol. 123, pp. 594-603, 042012. M3: Article.

[8] M. I. Lourakis and A. A. Argyros, "Sba: A software package for generic sparse bundle adjustment," ACM Transactions on Mathematical Software (TOMS), vol. 36, no. 1, p. 2, 2009.

[9] A. V. Bhavsar and A. Rajagopalan, "Towards unrestrained depth inference with coherent occlusion filling," International journal of computer vision, vol. 97, no. 2, pp. 167-190, 2012.

[10] R. D. Fiete, Modeling the imaging chain of digital cameras. SPIE press, 2010.

[11] K. Chang, RF and microwave wireless systems, vol. 161. John Wiley and Sons, 2004.

[12] H. J. Visser, Antenna theory and applications. John Wiley and Sons, 2012.

[13] R. S. Elliott, Antenna theory and design. Hoboken, N.J: Wiley-Interscience, 2003.

[14] J. M. Blackledge, Digital image processing: mathematical and computational methods. Horwood publishing, 2005.

[15] S. J. Orfanidis, Electromagnetic waves and antennas. Rutgers University New Brunswick, NJ, 2002.

[16] V. Antti and A. Lehto, Radio engineering for wireless communication and sensor applications. Artech House, 2003

[17] P. S. Cristobal Gabriel and H. Thienpont, Optical and Digital Image Processing: Fundamentals and Applications. John Wiley and Sons, 2011.

[18] D. J. Daniels, Antennas, pp. 83-127. Hoboken, NJ, USA: John Wiley and Sons, Inc.

[19] C. A. Balanis, Antenna theory: analysis and design. John Wiley and Sons, 2012. 